\title{
An Intelligent V2I-Based Traffic Management System
}

\author{
Vicente Milanés, Jorge Villagrá, Jorge Godoy, Javier Simó, Joshué Pérez, and Enrique Onieva
}

\begin{abstract}
Vehicles equipped with intelligent systems designed to prevent accidents, such as collision warning systems (CWSs) or lane-keeping assistance (LKA), are now on the market. The next step in reducing road accidents is to coordinate such vehicles in advance not only to avoid collisions but to improve traffic flow as well. To this end, vehicle-to-infrastructure (V2I) communications are essential to properly manage traffic situations. This paper describes the AUTOPIA approach toward an intelligent traffic management system based on V2I communications. A fuzzy-based control algorithm that takes into account each vehicle's safe and comfortable distance and speed adjustment for collision avoidance and better traffic flow has been developed. The proposed solution was validated by an IEEE-802.11p-based communications study. The entire system showed good performance in testing in realworld scenarios, first by computer simulation and then with real vehicles.
\end{abstract}

Index Terms-Data communication, road vehicles, traffic control, traffic management, vehicle-to-infrastructure (V2I) communication.

\section{INTRODUCTION}

A DVANCED driver-assistance systems (ADASs) have shown themselves to be an ideal tool for improving safety on the roads and, at the same time, reducing contamination and the magnitude of traffic jams, with the goal being greener and smarter driving. Examples of such systems are adaptive cruise control (ACC) based on radar technology [1] and traffic warning signals using artificial vision [2]. However, in common driving environments, there exist additional elements that need to be considered-other vehicles, pedestrians, emergency vehicles, motorbikes, cyclists, etc. With this in mind, a short-term goal is to design and implement cooperative systems based on communications that guarantee information exchange among these elements in the nearby environment.

There have been various approaches to solving this problem. Among them, the U.S. Department of Transportation promotes, under the IntelliDrive Initiative, the development of safety and mobility applications to identify possible crash scenarios and

Manuscript received March 9, 2011; revised June 27, 2011 and August 19, 2011; accepted September 17, 2011. Date of publication January 1, 2012; date of current version March 5, 2012. This work was supported by the CYCIT (Spain) and Plan Nacional (Spain) under Project GUIADE (P9/08) and Project TRANSITO (TRA2008- 06602-C03-01), respectively. The Associate Editor for this paper was L. Vlacic.

V. Milanés, J. Villagrá, J. Godoy, J. Pérez, and E. Onieva are with the Centre for Automation and Robotics, 28500 Madrid, Spain (e-mail: vicente.milanes@ csic.es; jorge.villagra@csic.es; jorge.godoy@csic.es; joshue.perez@csic.es; enrique.onieva@csic.es).

J. Simó is with the Department of Signal Theory and Communications, Universidad Rey Juan Carlos, 28943 Fuenlabrada, Spain (e-mail: javier.simo@urjc.es).

Color versions of one or more of the figures in this paper are available online at http://ieeexplore.ieee.org.

Digital Object Identifier 10.1109/TITS.2011.2178839 alert drivers through opportune visual or aural warnings. These applications are based on the communications standards defined for Wireless Access in Vehicular Environments (WAVE): IEEE 1609 and IEEE 802.11p. WAVE systems consist of three elements, i.e., the roadside unit (RSU), which is designed to be installed on traffic lights, signals, and other road elements; the onboard unit (OBU), which is designed to be mounted on the vehicles to guarantee connectivity; and the service channels $(\mathrm{SCHs})$, which allow bidirectional vehicle-to-vehicle $(\mathrm{V} 2 \mathrm{~V})$ or vehicle-to-infrastructure (V2I) connectivity [3].

In Asia, the Universal Traffic Management Society of Japan has been working on Driving Safety Support Systems (DSSSs) that would alert drivers to potential dangers on the road [4], [5]. These systems are based on dedicated short-range communication (DSRC) and infrared V2I communications. Several real tests demonstrated the effectiveness of the first DSSS at intersections. Another major program is the Ubiquitous ITS initiative, which is part of the e-Japan Strategy. This program is implemented in $\mathrm{V} 2 \mathrm{~V}$ communications systems using two radiofrequency (RF) channels: one in the band between 5811.5 and $5828.5 \mathrm{MHz}$ for communications and the other between 669 and $679 \mathrm{MHz}$ for control signals [6]. In 2008, DENSO tested these systems on public roads of Abashiri, Hokkaido, Japan, to measure the noise in the communications while vehicles are moving.

In Europe, the objective of the Cooperative Vehicle Infrastructure Systems project is to design and develop the elements needed for continuous and transparent communication between vehicles and infrastructure based on the Communication Access for Land Mobiles ISO standard (CALM). This architecture allows V2V and V2I communications through various access technologies: second- and third-generation cellular networks, infrared, WiFi (IEEE $802.11 \mathrm{a} / \mathrm{b} / \mathrm{g}$ ), or WAVE [7]. In the same research line, the SAFESPOT project is aimed at improving road safety by combining the information incoming from vehicles and infrastructure into local dynamics maps [8]. With these maps, safety applications will be able to detect critical situations in advance, thereby improving response times. Another project is that of the Co-operative Systems for Intelligent Road Safety (COOPERS), which aims to maintain continuous bidirectional V2I communications, allowing vehicles to exchange relevant data for a specific segment of the road to improve safety and enable efficient traffic management [9], [10].

Since all these cooperative applications are based on effective communications, it is necessary to study their performance under real critical conditions, in which a great amount of information is exchanged through the network due to the high number of vehicles present in the near environment. 


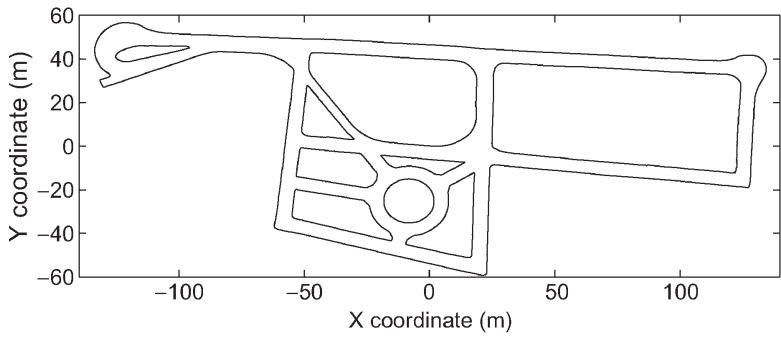

Fig. 1. AUTOPIA driving area.

Various experiments and simulations have studied different test scenarios: communications delays due to the number of nodes, channel noise, and connection failures between nodes [11]-[15].

This paper describes an intelligent V2I-based traffic management system. The goal is to coordinate traffic in a limited urban area, in which different driving scenarios can coexist (see Fig. 1). A control station is in charge of evaluating the traffic conditions to prevent collisions well in advance and improve traffic flow. To this end, a Driving state indicator-representing a tradeoff between safety and fluidity in driving-is sent to the drivers with a recommended action to adjust the vehicle's direction and speed toward an optimal state. Given the key role that communications play for the operation of this system, an evaluation of their requirements was carried out. The performance of the V2I management system was tested in simulations and in an experiment using real cars on a test circuit.

With respect to this study's place in the ongoing AUTOPIA program, it introduces an intelligent traffic management system that is capable of analyzing all the information in its vicinity and sending the recommended action to the vehicles and presents the first AUTOPIA experiment with four real vehicles. The main contributions of this paper may be summarized as follows:

1) an intelligent evaluation system to determine how drivers are managing their vehicles and sending them optimal reference targets;

2) a communications study based on the IEEE 802.11p standard to demonstrate the feasibility of the proposed solution;

3) an experimental study - both in simulations and on a real road - to validate the proposed system with four vehicles on the test track aimed at determining how drivers manage the information coming from the local control station.

\section{Statement of the Problem}

Congestion in large towns is one of the principal problems faced by transportation systems. Our approach to a solution is based on the use of control stations, each of which manages the traffic within its area of influence. These stations need to be coordinated to resolve conflicts at their borders. This section describes the missions that the local station has to carry out and the requirements for the different stretches of road into which its area of influence is divided. This study focuses on an isolated local control area-communication among different local control areas is beyond its scope, although an idea of how this problem may be solved is presented in [16].
Fig. 1 shows the test track modeling a typical urban area that will be used in the experimental phase. It includes a roundabout, $\mathrm{T}$ - and $\mathrm{X}$-shaped intersections, incorporations, $90^{\circ}$ bends, U-turns, and straight stretches. A local control station that is capable of receiving all the information coming from either the infrastructure or the vehicles has to be capable of intelligently managing the traffic to avoid collisions. This station receives and analyzes the information coming from the vehicles to send each driver information about how they are driving and an alert and recommended action to avoid any critical situation. In particular, the control station is responsible for determining the Driving state on the basis of the vehicle's location, direction, speed, and the road layout.

Once the control station has received information from the vehicles, its responsibilities are given as follows:

1) to classify the vehicles within the driving area;

2) to identify stopped vehicles as obstacles;

3) in straight stretches, to inform each vehicle about its vicinity, i.e., whether there exists a leading vehicle that affects the current speed and whether overtaking is possible;

4) in bend stretches, to provide information in advance about the road layout;

5) to manage each specific area using priority levels according to the kind of vehicle to avoid potential collisions;

6) to identify in advance traffic situations of risk.

Assuming that all the vehicles are equipped with a communications system, there are two problems that have to be tackled. 1) Is the control station capable of managing all the vehicles that may be driving within its control area? 2) How should the traffic be managed to avoid collisions and improve traffic flow? Respective solutions to these two questions will be presented in the two succeeding sections.

\section{VehicLE-TO-INFRASTRUCTURE-BASED SOLUtion}

As previously noted, a control station is responsible for managing and processing information coming from the vehicles and for returning warning and recommendation commands to the vehicles to improve safety and traffic flow. This information exchange between the control station and the vehicles clearly has to be as reliable as possible.

\section{A. Data Structure}

We defined two data package structures for the information exchange. Table I lists the fields to send and their size. From the vehicles to the infrastructure (see the left-hand side of Table I), the fields considered correspond to an identification number for each kind of vehicle, a timestamp, vehicle positioning (two fields), speed, and a space reserved for future variables that may be interesting to transmit, e.g., the vehicle's intentions. From the infrastructure to the vehicle (see the right-hand side of Table I), the fields to send are the vehicle identification number, two fields related to longitudinal actions, two fields related to lateral actions, and a space reserved for future applications, e.g., road layout or weather. The longitudinal actions correspond to the throttle and brake pedals. The first field is used to send 
TABLE I

Proposed Communications Data PACKages

\begin{tabular}{|c|c|}
\hline \multicolumn{2}{|c|}{ Vehicle-to-Infrastructure } \\
\hline Field & Type (Size) \\
\hline Vehicle ID & Short (2B) \\
\hline Timestamp & U Integer (4B) \\
\hline Northing & Double (8B) \\
\hline Easting & Double (8B) \\
\hline Speed & Float (4B) \\
\hline Reserved & $\mathrm{X} \mathrm{(6B)}$ \\
\hline
\end{tabular}

\begin{tabular}{|c|c|}
\hline \multicolumn{2}{|c|}{ Infrastructure-to-Vehicle } \\
\hline Field & Type (Size) \\
\hline Vehicle ID & Short (2B) \\
\hline Speed warning & Short (2B) \\
\hline Speed control & Short (2B) \\
\hline Steering warning & Short (2B) \\
\hline Steering control & Short (2B) \\
\hline Reserved & X (6B) \\
\hline
\end{tabular}

a warning signal to the driver as a function of the Driving state. The second is used to send a recommended speed to avoid collisions or improve traffic flow. This second field can autonomously act on the vehicle if the latter is equipped with automated actuators. The next two fields have similar functions, i.e., warning and recommendation, but now corresponding to lateral control, i.e., the steering wheel.

\section{B. Analysis of the Communications System}

The proposed intelligent traffic system is based on having reliable V2I communications. The following paragraphs will describe the solution adopted and an analysis of its capacity to support reliable information exchange in the face of delays and packet losses.

The V2I-based intelligent management traffic system uses WAVE for the V2I communications. WAVE is based on the IEEE $802.11 \mathrm{p}$ standard for physical layer (PHY) and medium access control (MAC), and on additional standards of the IEEE 1609 family that deal with logical link control, multichannel management, security, and other issues. IEEE $802.11 \mathrm{p}$ is an amendment to the IEEE 802.11-2007 standard that proposes enhanced distributed channel access (EDCA) for the MAC and a PHY similar to IEEE 802.11a but using channels of $10 \mathrm{MHz}$. EDCA defines four different traffic classes or "access categories" that are given different priorities: 1) AC_VO (Voice Access Category); 2) AC_VI (Video Access Category); 3) AC_BE (Best-Effort Access Category); and 4) AC_BK (Background Access Category). Depending on the priority assigned to a given packet, it will be queued for the corresponding access category (see [17] for details). Another important characteristic of WAVE is its multichannel feature. There are several $10-\mathrm{MHz}$ channels in the DSRC band that can be used. One is the control channel $(\mathrm{CCH})$, and the other channels are SCHs. All the stations-vehicles-must listen to the $\mathrm{CCH}$, in which packets can be sent and received without any prior association, but only stations that have joined a wireless basic service set (WBSS) may exchange packets over an associated SCH. Time is slotted into 100-ms superframes, each one starting with about $50 \mathrm{~ms}$ of contention in the $\mathrm{CCH}$, followed by a similar time in which stations belonging to a WBSS may exchange packets in an SCH. In summary, WAVE has introduced into the IEEE 802.11 standard all the improvements that make efficient and stable communications possible at normal road speeds without any significant performance drop relative to static communications (see [3] and [18] for good tutorials about WAVE).

Regarding the use of EDCA, Mora et al. [19] demonstrated that, under heavy load conditions-even using traffic differentiation-real-time traffic may suffer from unacceptable packet losses and high delays. They also suggest that dynamic adjustments of the contention window size for all access categories can significantly improve the performance, as also had been demonstrated by [20].

A scenario very similar to ours is studied by Bohm and Jonsson [21], but they avoid contention for the channel by implementing a polling mechanism. As we shall show here, standard EDCA appears to be sufficient for our application to handle critical traffic under heavy load conditions, but Bohm and Jonsson's proposal would be reasonable in other more complex scenarios.

Although the preceding comments seem to suggest that EDCA may not be ideally suited to our application, the values proposed for the EDCA parameters in $802.11 p$ have a very special quality: when the AC_VI access category is not used, AC_VO is not only highly prioritized but also deterministically protected from any possible collision with AC_BE and AC_BK traffic, at least at the first transmission attempt. Even in the case of collisions among AC_VO packets, retransmissions still get very high priority. As long as the number of stations transmitting AC_VO traffic is kept low, delay and packet loss are kept under certain limits for that prioritized traffic, regardless of what happens with the less-prioritized traffic classes [21]. Hence, if only the RSU and a very few vehicles in special situations (emergencies, breakdown vehicles, etc.) are allowed to use $\mathrm{AC}$-VO traffic, and $\mathrm{AC} \_\mathrm{VI}$ is generally unused, one can be sure that vehicles will always receive real-time packets from the infrastructure. The problem will then be how to keep the delay and packet-loss probability below certain limits for uplink traffic, whose intensity will depend on the number of vehicles within the coverage area.

For critical message dissemination, Eichler [22] suggested that a higher layer mechanism must be built on top of the WAVE stack to manage the network in such a way that the number of vehicles contending for the channel in this kind of scenario never exceeds a certain limit that must be carefully measured. With this premise, we here assumed that all vehicles send packets as described in the previous section every $100 \mathrm{~ms}$, and then, we determined the number of vehicles representing the threshold beyond which delay and packet loss grow too much. To estimate the delay and packet loss, we simulated our scenario with one RSU, i.e., the traffic control station, and an increasing number of vehicles, using the WLS EDCA simulator ${ }^{1}$. All simulations used the values of the parameters listed in Table II.

The results of the simulations ${ }^{2}$ clearly show, as expected (see Fig. 2), how the RSU packets maintain the quality of service (QoS), even with a very high number of vehicles. Lessprioritized traffic coming from vehicles, however, maintains low delays and insignificant collision probabilities only when there are fewer than 40 stations. Beyond that limit, as the

\footnotetext{
${ }^{1}$ The WLS simulator [17] was developed to exactly implement EDCA. It has been extensively validated by the authors and by other researchers.

${ }^{2} \mathrm{RSU}$ sending short AC_VO packets every $50 \mathrm{~ms}$ and a minimum of 20 vehicles sending AC_BE traffic.
} 
TABLE II

PARAMETERS FOR SimUlations AND EXPERIMENTS With EDCA

\begin{tabular}{|c|c|}
\hline Parameter & Assigned value \\
\hline Channel Width & $10 \mathrm{MHz}$ \\
\hline MACHeader & 232 bits \\
\hline PHY Overhead & 13 symbols $(8 \mu \mathrm{s} / \mathrm{sym} \mathrm{mol})$ \\
\hline BasicBitRate & $3 \mathrm{Mbps}$ \\
\hline BitRate & $6 \mathrm{Mbps}$ \\
\hline Propagation Time & up to $2 \mu \mathrm{s}$ \\
\hline SIFS & $32 \mu s$ \\
\hline Slot Time & $13 \mu s$ \\
\hline$A I F S N_{0}\left(A C_{-} V O\right)$ & 2 \\
\hline$A I F S N_{2}\left(A C \_B E\right)$ & 6 \\
\hline$A I F S N_{3}\left(A C \_B K\right)$ & 9 \\
\hline$C W_{\min , 0}$ & 4 \\
\hline$C W_{\max , 0}$ & 8 \\
\hline$C W_{\min , 2}$ & 16 \\
\hline$C W_{\max , 2}$ & 1024 \\
\hline$C W_{\min , 3}$ & 16 \\
\hline$C W_{\max , 3}$ & 1024 \\
\hline WSMP Header & 11 bytes \\
\hline
\end{tabular}
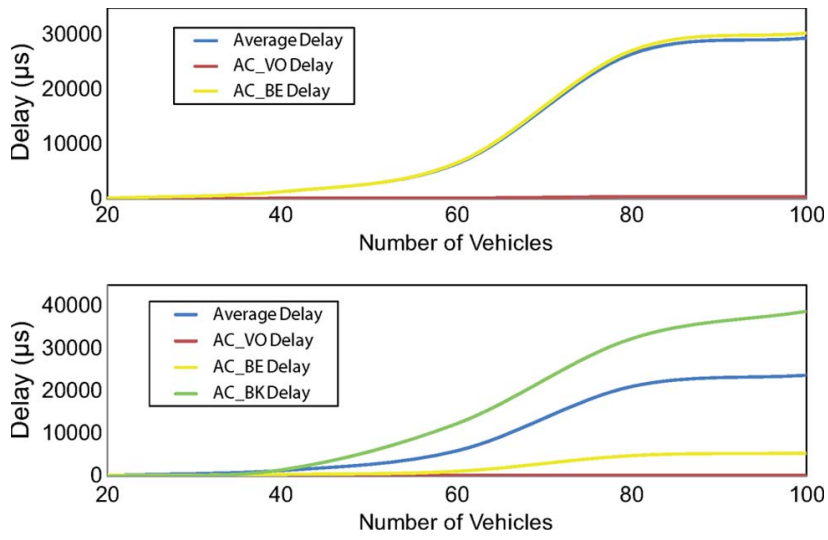

Fig. 2. (a) One RSU and a variable number of vehicles sending one packet/superframe each. (b) One RSU, 20 vehicles with AC_BE traffic, and a variable number of vehicles with AC_BK traffic sending one packet/superframe each.

number of vehicles generating traffic increases, the QoS very rapidly worsens.

These results imply that an application-level mechanism that maintains a low number of stations contending for the safetycritical traffic channel has to be proposed. This is not at all necessary for the experiment that we shall present in the succeeding sections, but the problem will have to be theoretically solved for our proposals to be generally applicable. Considering WAVE specifications, the RSU can announce a WBSS during the $\mathrm{CCH}$ so that stations can join it in an SCH just by internally deciding to do so. Invitations for the WBSS may be either generally broadcast packets with information that permits the stations to decide whether to join it or not, or unicast packets if necessary. As opposed to the $\mathrm{CCH}$, traffic in the WBSS during the $\mathrm{SCH}$ comes only from the RSU and from those vehicles that have joined the WBSS. The packet protocol used in the $\mathrm{CCH}$ is WSMP (which is more suitable than its alternative, i.e., IPv6), and it would also be used for the WBSS for efficiency.

Hence, if, during the $\mathrm{CCH}$, the RSU invites certain vehicles to join a safety-critical WBSS, with either broadcast packets or packets unicast to certain vehicles, a limited number of vehicles may exchange traffic on the $\mathrm{SCH}$ with no interference from the rest of the vehicles. The high-level application in the vehicles is responsible for observing packets sent during the $\mathrm{CCH}$, which inform about the coordinates and size of any critical area and use that information and their own coordinates to decide whether they should join the announced safety-critical WBSS in the SCH. Using this solution, we remain fully within the limits of the standards, and our simulation results show that packet-drop probabilities and delays are kept below reasonable levels (see Table III).

The simulations also verified that the number of $\mathrm{AC}_{-} \mathrm{VO}$ transmissions may be ten times or more greater than expected with no significant increase in the AC_VO packet-drop probability and delay. For up to ten stations, each producing the same amount of traffic as the RSU, the packet-loss probability remains null, and the average delay with up to 100 stations contending for the channel stays at $515 \mu \mathrm{s}$. This illustrates that our system tolerates the presence of some emergency vehicles in the area using $\mathrm{AC}_{-} \mathrm{VO}$ in the $\mathrm{CCH}$ with very low impact on the QoS.

The performance limits have only been analyzed from the perspective of the QoS offered to safety-critical traffic and not from that of the computing and communications capacities of the RSU. This is because the limits imposed by the QoS requirements are almost one order of magnitude more stringent than any RSU capacity constraints. The RSU is not significantly more complex than a custom 802.11e EDCA access point, with the exception of the multichannel RF hardware block and the corresponding control algorithms. Indeed, most of the traffic taking place in a connectionless fashion reduces the complexity of the computational tasks at the RSU. With respect to the communications limits, the last two rows in Table III show that the greatest channel occupation foreseen is slightly more that $10 \%$ and the maximum throughput is still extremely low. Hence, the only really significant restrictions in our scenario are those corresponding to the QoS. The performance analysis only considers a high number of vehicles that can be covered by a single RSU at low speeds. The reason is that, as the average speed of the vehicles increases, the distances between them also increase, so that fewer vehicles are covered by the RSU, and the intensity of the communications traffic sharply falls.

For the experimental part of this work that will be described in Section V, 802.11p was hardware emulated with 802.11a radios by appropriately adjusting various parameters (see Table II). However, because so few vehicles were involved in the experiment, the old 802.11e EDCA, simple contention in the $\mathrm{CCH}$, or the complete solution proposed here are all valid options.

\section{Traffic Management System}

Having shown the feasibility of the proposal from the communications perspective, we next consider the other question to be resolved-how to avoid collisions and improve traffic flow.

The traffic control station has to manage all the information coming from the vehicles and, when the environment requires it, return a warning signal with the Driving state and a recommended action. A detailed description of its responsibilities was presented in Section II. The fields to be sent by the control 
TABLE III

Mean Delay, Standard Deviation of Delay, and PaCKet-Loss Probability for Lower Priority Traffic

\begin{tabular}{|c|c|c|c|c|c|c|}
\hline $\begin{array}{l}\text { N. } A C \_B E \text { flows } \\
\text { N. } A C_{-} B K \text { flows }\end{array}$ & $\begin{array}{r}20 \\
0\end{array}$ & $\begin{array}{r}40 \\
0\end{array}$ & $\begin{array}{r}60 \\
0\end{array}$ & $\begin{array}{l}20 \\
20\end{array}$ & $\begin{array}{l}20 \\
40\end{array}$ & $\begin{array}{l}20 \\
60 \\
\end{array}$ \\
\hline$A C \_B E$ Delay (Mean) & $213.00 \mu s$ & $437.03 \mu s$ & $1613.23 \mu s$ & $408.45 \mu s$ & $1111.8 \mu \mathrm{s}$ & $4975.35 \mu s$ \\
\hline AC_BE Delay (Standard Deviation) & $2.44 \mu s$ & $14.69 \mu \mathrm{s}$ & $152.57 \mu s$ & $9.49 \mu s$ & $58.41 \mu s$ & $142.32 \mu s$ \\
\hline$A C \_B K$ Delay (Mean) & r. & - & - & $562.26 \mu \mathrm{s}$ & $2538.70 \mu s$ & $32546.49 \mu \mathrm{s}$ \\
\hline$A C \_B K$ Delay (Standard Deviation) & & & & $19.71 \mu s$ & $179.97 \mu s$ & $335.89 \mu s$ \\
\hline Packet-loss Prob. & 0 & 0 & 4.97E-4 & 0 & 0 & 0.364 \\
\hline Busy channel (\%) & 2.778 & 5.445 & 8.112 & 5.445 & 8.112 & 10.778 \\
\hline Throughput (Mbps) & 0.052 & 0.104 & 0.154 & 0.104 & 0.154 & 0.206 \\
\hline
\end{tabular}

station were listed in the right-hand part of Table I. For the sake of simplicity, this study deals only with the longitudinal actions, so that only recommended speeds are sent to the vehicles.

With these premises, the two reserved 2-byte fields, i.e., Speed warning and Speed control, are used to send the Driving state and the recommended speed for each car, respectively. Specifically, the Speed warning signal is used to codify the Driving state in the interval $[-1,1]$. With respect to the Speed control, this field is responsible for sending the reference speed that is the best driving action for each driver to take. If the vehicle is equipped with automatic systems (ACC capabilities for longitudinal control), this command can be automatically applied.

\section{A. Management Solution}

The main difficulty arises from the fact that the control station has to be capable of properly managing all the traffic circumstances safely. Our approach is based on recent results presented in [23], following the previous outline by the partners of the Advanced Transportation TecHnology (PATH) program (see Lu et al. [24]) based on magnetic markers.

It consists of a projection of the vehicles onto the same lane, independently of which driving situation they are involved in, i.e., all the vehicles are considered to be involved in a virtual ACC system. Thus, if four vehicles are approaching an intersection, they are projected onto the same lane and treated as a platoon so that a recommended distance and speed can then be generated for each of them. With this projection, both the Driving state and the recommended speed can be sent to each car. The same reasoning is applicable to any traffic situation (see [23] for details).

With this procedure and once the priority has been assigned to each car according to Spain's Road Circulation Code or following some basic criterion on the part of the control station (e.g., public transport vehicles have priority over private vehicles), the speed and distance references can be generated by the management system to generate the recommended speed and to be used as input by the control system.

\section{B. Generation of the Reference Speed and Distance}

The goal of the traffic management system will be to coordinate vehicles to track as precisely as possible a reference distance between vehicles $d_{r}$ and a target relative speed $v_{r}$.

Some approaches [25], [26] have tried to reproduce human behavior to achieve a "comfort-based" reference interdistance (or spacing). Unfortunately, this kind of strategy may not necessarily lead to safe operation. Other researchers (e.g., [27] and
[28]) have modeled the reference interdistance using different types of time polynomials, whose coefficients are obtained by applying safety acceleration and jerk constraints. These approaches, in general, yield acceptable results in an ACC, but when the preceding car suddenly decelerates, the vehicles present a transitory relative speed that is too great. To deal with this problem, we used a dynamic reference model [29] to generate the two variables required (see [30] or [31] for recent implementations using this model).

The reference distance is related to the safe nominal interdistance $d_{0}$ (which is the maximum distance at which the control algorithm will be activated) and the critical distance $d_{c}$ (which is the minimum distance between cars, which is only attained when they are stopped). Note that the dynamic reference model used in this work will yield a smaller reference interdistance than the classical 2-s distance rule, which is moreover extremely complex to compute and handle at low speeds.

The interdistance reference model is expressed as follows:

$$
\begin{aligned}
& v_{r}=\frac{c}{2}\left(d_{0}-d_{r}\right)^{2}+\dot{x}_{l}(t)-V_{\max } \\
& \dot{d}_{r}=v_{r}
\end{aligned}
$$

where $\dot{x}_{l}$ is the preceding vehicle's speed, $V_{\max }$ is the maximum allowable speed, and $c$ is a design parameter. Equation (1) describes the dynamics of a virtual vehicle, which is positioned at distance $d_{r}$ (the reference distance) from the leading vehicle, such that the following comfort and safety constraints are fulfilled.

1) $d_{r} \geqslant d_{c}$, with $d_{c}$ being the minimal intervehicle distance.

2) $\left|\ddot{x}_{r}\right| \leqslant \gamma_{\max }$, where $\gamma_{\max }$ is the maximum attainable longitudinal acceleration.

3) $\left|\dddot{x}_{r}\right| \leqslant J_{\max }$, with $J_{\max }$ being a bound on the driver's desired jerk.

Note that this reference velocity depends on the leading vehicle's distance $d_{0}$ and parameter $c$, which is, in turn, an algebraic function of the safety and comfort parameters $d_{c}$, $V_{\max }, \gamma_{\max }$, and $J_{\max }[29]$.

\section{Driving State}

A fuzzy traffic management system was developed to evaluate the traffic situation. Fuzzy logic is considered a good control technique when dealing with systems whose definition is imprecise and has been extensively applied in industrial applications in recent years [32]. In this case, the control station, of course, has to advise the drivers about how they are driving, but how is this to be quantified in each case? 


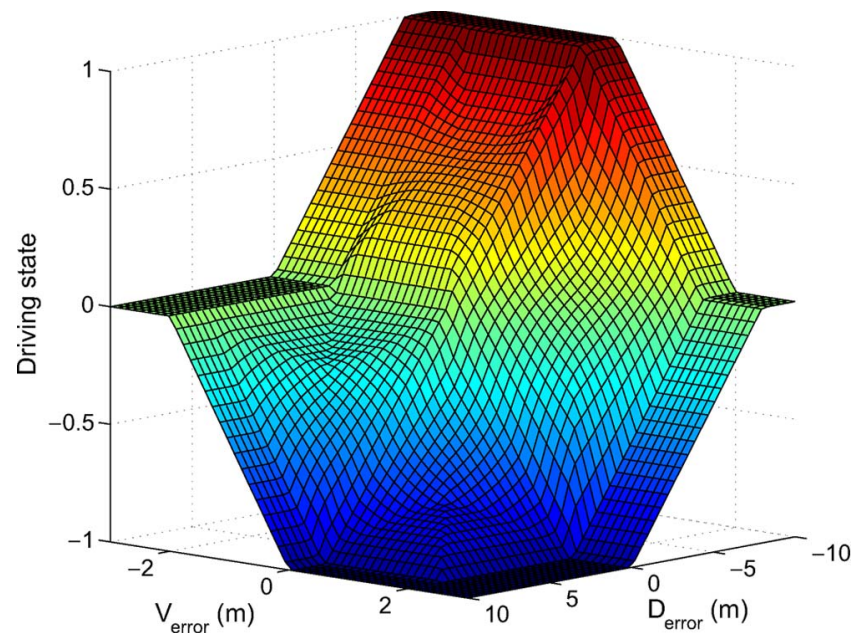

Fig. 3. Control surface to evaluate the level of risk.

With the information available at the control station, the actual and reference distances between vehicles and the actual and recommended speeds for a vehicle involved in a traffic situation are known. The errors in the distance $\left(D_{\text {error }}\right)$ and the speed $\left(V_{\text {error }}\right)$ are then used as inputs to the fuzzy controller. As output, the Driving state is sent to the vehicles. The output of the controller is codified using three singletons located at $-1,0$, and 1 , representing safe driving but poor traffic flow, an optimal tradeoff between safety and traffic flow, and a high risk of collision, respectively.

Note that, since the system previously described was developed to be applied in urban environments, the maximum allowed speed is set at $50 \mathrm{~km} / \mathrm{h}$.

Three symmetrical triangular membership functions are defined for each input variable, i.e.., the errors with respect to the reference distance and speed that are evaluated to determine the Driving state. For the speed, the triangles have centers situated at $-2,0$, and $2 \mathrm{~m} / \mathrm{s}$, representing membership functions denominated the High_risk, Optimal, and Low_fluid driving state.

Similarly, the triangles defined to codify the distance have centers at $-8,0$, and $8 \mathrm{~m}$, which are denominated as in the previous case as High_risk, Optimal, and Low_fluid driving state. Values outside the range ( \pm 2 for the speed and \pm 8 for the distance) are assigned a degree of membership for each corresponding membership function. The control surface generated by the fuzzy controller is shown in Fig. 3.

The choice of the membership functions was based on the driving experience of human drivers, particularly that of the authors. However, the actual values of these functions were experimentally adjusted to our real vehicles on the test tracks. These tests showed that these membership functions were suitable for the problem at hand. To check the controller in traffic circumstance that were impossible to reproduce in our facilities, we used the simulator that we shall describe in Section V-A. The results of the simulations justified the choice of the membership functions.

The fuzzy traffic management system implemented in the local control station is designed to obey Spain's Road Circulation Code. Hence, if vehicles are driving in an area without
TABLE IV

COMMUNICATIONS RESULTS FOR THE EXPERIMENTS

\begin{tabular}{|c|c|}
\hline & 4 vehicles \\
\hline Service Time (mean) [ $\mu s]$ & 482.19 \\
\hline Service Time (std) $[\mu s]$ & 85.76 \\
\hline Service Time $(\min )[\mu s]$ & 424.67 \\
\hline Service Time (max) $[\mu s]$ & 1336.91 \\
\hline Packet-collision probability & 0.002 \\
\hline Packet-drop probability & $\sim 1 \mathrm{E}-019$ \\
\hline
\end{tabular}

traffic signals, the drivers do not need to know which vehicle has priority since they receive already processed information about how they are driving through this area and which speed is appropriate to drive with safety while improving traffic flow.

\section{Results}

In this section, we shall present some of the test results in both the simulations and the real environment. For these tests, an intersection was selected as the region of interest since it represents the most difficult scenario in which the V2I-based traffic management system will have to work. The particular scenario analyzed is one in which four vehicles are simultaneously approaching the intersection. Assuming that the intersection is not signalized and three private vehicles and a bus are approaching the crossroad, the highest priority is assigned to the bus and the priorities of the other vehicles are assigned following the Road Circulation Code. The communication system's behavior in each case for this scenario is presented in Table IV, including the packet-collision and packet-drop probabilities.

Compared to Table III, Table IV lists service times. These include the MAC and propagation delays. as well as the queuing times, confirmation times, and retransmission overheads. They are given to better approximate the high-level latency of the communications system, as perceived by the applications exchanging the traffic. It is observed that the service time is extremely stable; even the highest value obtained in the experiments has null impact on the quality of the system. The packet-collision probability is given to show that, in this experimental scenario with only four vehicles, such collisions are very scarce, and retransmissions make it virtually impossible to lose a packet, as evidenced by the value of the packet-drop probability obtained by simulation. These values show that communications are not stressed in this experiment. Indeed, the fact that there were no communication errors matched the expectation.

Note that, to test the traffic management system under different conditions, the simulation and experimentation speeds were significantly different. The test scenario with real vehicles was restricted by the size of the Center for Automation and Robotics (CAR's) facilities-the maximum speed was therefore limited to $10 \mathrm{~km} / \mathrm{h}$. The simulated maneuvers, however, were tested at the maximum speed allowed in urban areas, i.e., $50 \mathrm{~km} / \mathrm{h}$.

\section{A. Simulation Experiment}

The traffic management system was implemented on a simulator developed ad hoc on the Matlab/Simulink platform, with the dynamics of four vehicles recreated using the model detailed in [33]. The same longitudinal proportional-differential 

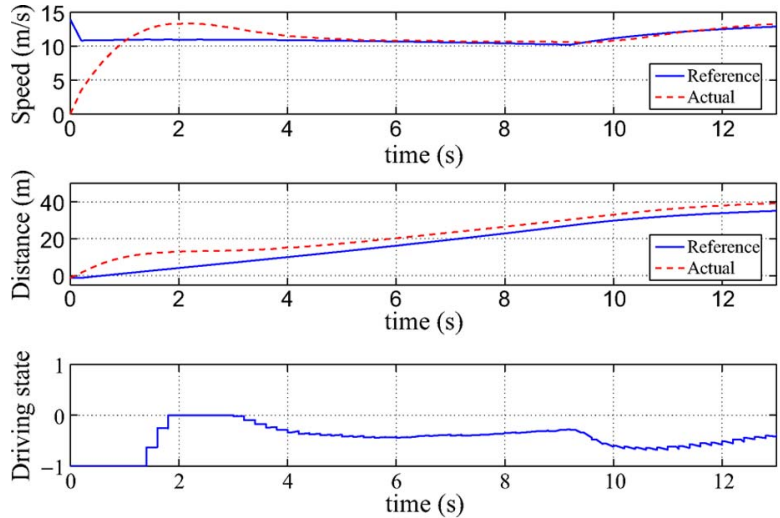

Fig. 4. Simulation results: second vehicle in terms of right-of-way approaching the intersection.

controller, i.e., $K_{p}=0.3$ and $K_{i}=0.1$, is used in each vehicle to track the desired speed. In addition, in each vehicle, the GPS positioning and vehicle measurements are artificially perturbed with a white Gaussian noise. The sampling rate is constrained by the GPS frequency $\left(T_{s}=0.2 \mathrm{~s}\right)$.

The variables $x$ that are used in the V2I communications are modeled as

$$
\begin{aligned}
& x\left(t_{k}\right)=x\left(t_{k}-\tau\right)+u\left(t_{k}\right) \\
& \qquad \begin{cases}u\left(t_{k}\right)=-x\left(t_{k}-\tau\right)+x\left(t_{k-1}-\tau\right), & \text { if } p\left(d<d_{c}\right) \\
u\left(t_{k}\right)=0, & \text { if } p\left(d \geqslant d_{c}\right)\end{cases}
\end{aligned}
$$

where $\tau$ is the service time, $d$ is the packet-drop probability, and $d_{c}$ is the critical value, whose values are given in Table IV.

To illustrate the algorithm's ability to evaluate the Driving state of each vehicle, a scenario in which four vehicles approach an X-shaped intersection was implemented. In the simulation, all four vehicles track a straight-line path, starting at similar distances from the intersection point. Consequently, the traffic management system must handle not only the priority order but also the manner in which each vehicle will safely cross the intersection. Therefore, a recommended speed and distance to the preceding vehicle has to be provided to the three vehicles, following the public transport bus, which is the vehicle with the highest priority.

The first vehicle runs at a constant speed, i.e., $50 \mathrm{~km} / \mathrm{h}$. The second tracks the recommended speed sent by the control station. The third simulates aggressive manual driving, such that the vehicle's speed is always above the reference. Finally, the fourth always runs below the target speed, emulating conservative manual driving.

Figs. 4-6 show the evolution of the speeds and the distances between vehicles-both the reference and actual values-for the second, third, and fourth vehicles, respectively.

The vehicle in Fig. 4 is autonomously driving, and the speed and distance targets are tracked well. Note that, even though the speed error is almost negligible, there is a constant distance error throughout the maneuver. As a result, the Driving state is always slightly under 0 . This value can be seen as reflecting a good tradeoff between safety and smooth traffic flow but giving slightly more priority to collision avoidance.
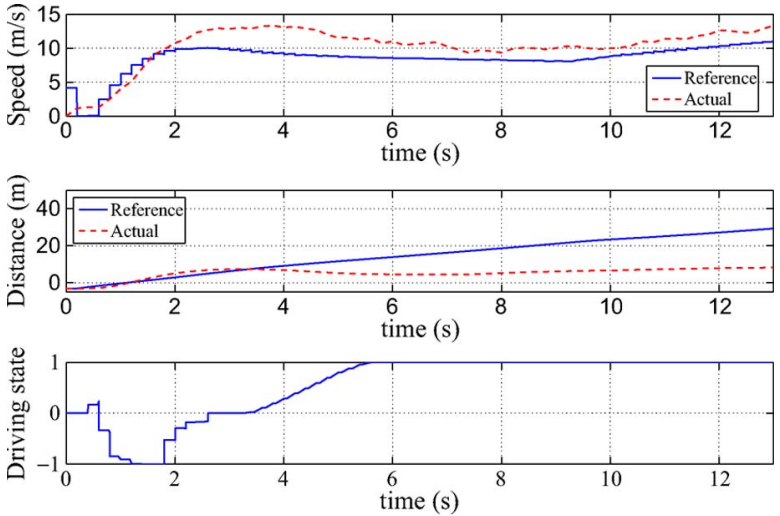

Fig. 5. Simulation results: third vehicle in terms of right-of-way approaching the intersection.
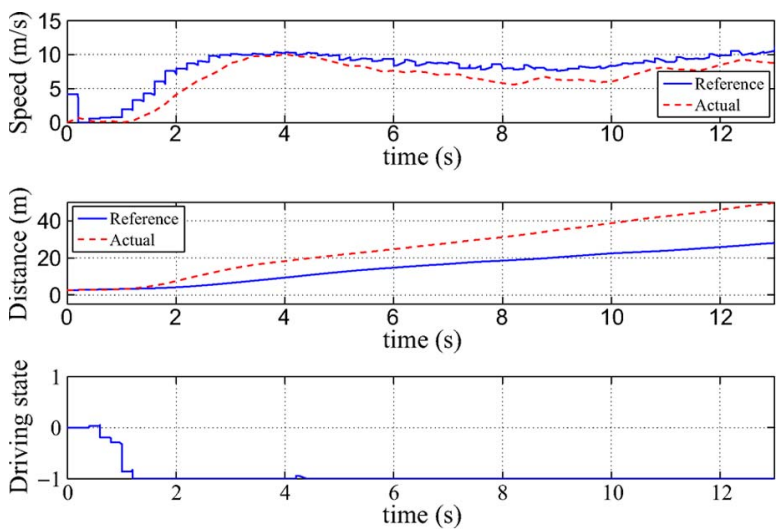

Fig. 6. Simulation results: fourth vehicle in terms of right-of-way approaching the intersection.

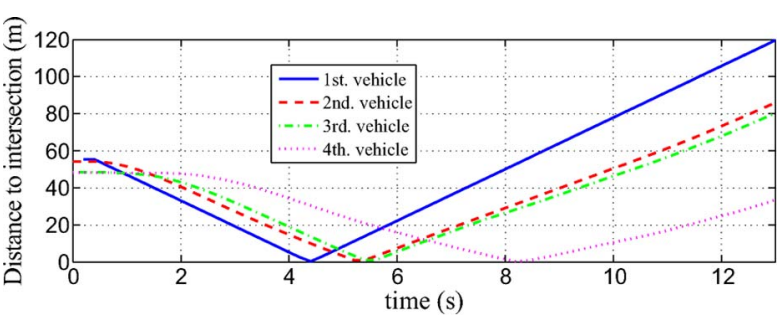

Fig. 7. Simulation results: distance to the intersection point for each vehicle.

In Fig. 5, the recommended speed is always exceeded, so that the tracking distance error monotonically increases until the vehicle crashes into its predecessor. This situation is reflected in the bottom plot of the figure, where the Driving State is estimated to be 1 from instant $t=5.4 \mathrm{~s}$ onward, which is the moment of the actual collision (see also Fig. 7).

Finally, Fig. 6 shows that the conservative behavior of the last vehicle leads to a Driving state that is equal to 0 from instant $t=1.2 \mathrm{~s}$. In this respect, Figs. 7 and 8 show that, at about $t=$ $9 \mathrm{~s}$, while the first three vehicles have ended their maneuver and are at a considerable distance from the intersection point, the last vehicle has barely passed through.

The evolution of the positions of the four vehicles is plotted in Fig. 8, in which one readily deduces that a crash would have occurred between the second and third vehicles. 


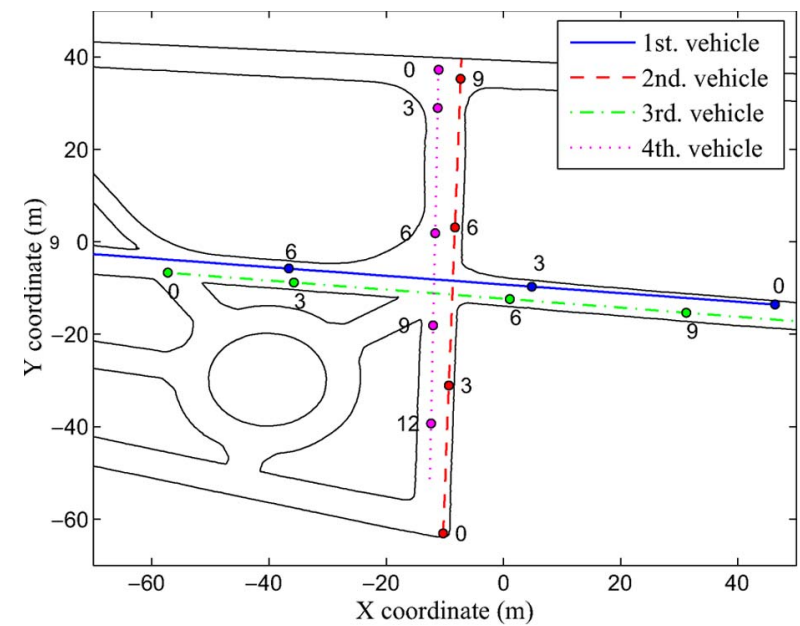

Fig. 8. Vehicles' positions in the simulation.

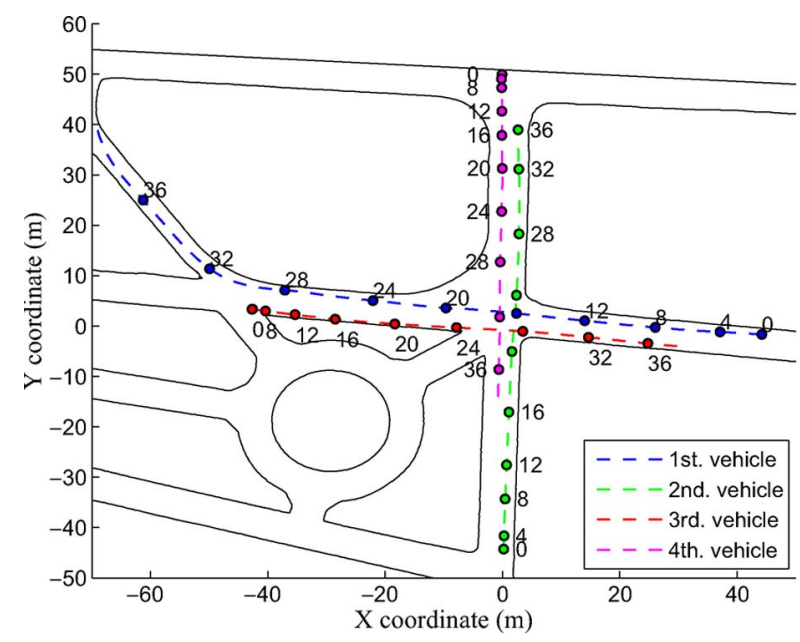

Fig. 9. Vehicles' positions during the experiment.

\section{B. Real Results at a Crossroad: The Study of One Case}

Trials using real vehicles were performed on the private driving circuit at CAR's facilities (see Fig. 1). The central intersection was used. Since only four vehicles were available for this test, the input coming from the roundabout was neglected. The goal was to evaluate in a real environment the capability of the control station to manage a traffic situation in which four vehicles coincide at a crossroad.

Description of the Vehicles: Four different vehicles were used. Two are fully automated gasoline-propelled production cars, with one of them being a convertible. The others are an electric van and an electric minibus. To make the experiments as close as possible to vehicles on the market, only warning signals are allowed, and the drivers can monitor the collision probability and recommended speed via a head-up display. Details of the vehicles' equipment and capacities can be found in previous work [23], [34]-[36].

Experimental Results: The real scenario is identical to that presented in the simulation results. Fig. 9 shows the evolution of the vehicles' positions during the experiment, and Fig. 10 shows their distances to the intersection point.

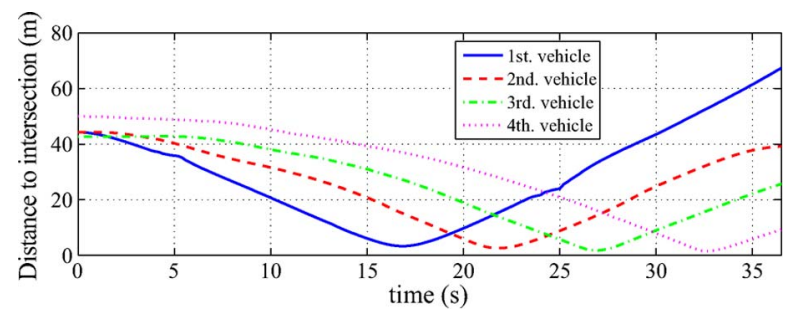

Fig. 10. Distances of the vehicles to the intersection point.
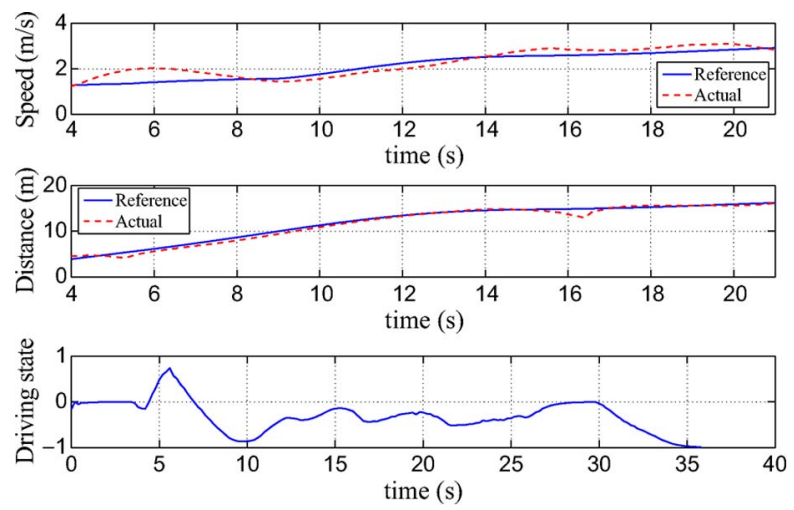

Fig. 11. Second vehicle in terms of right-of-way approaching the intersection.
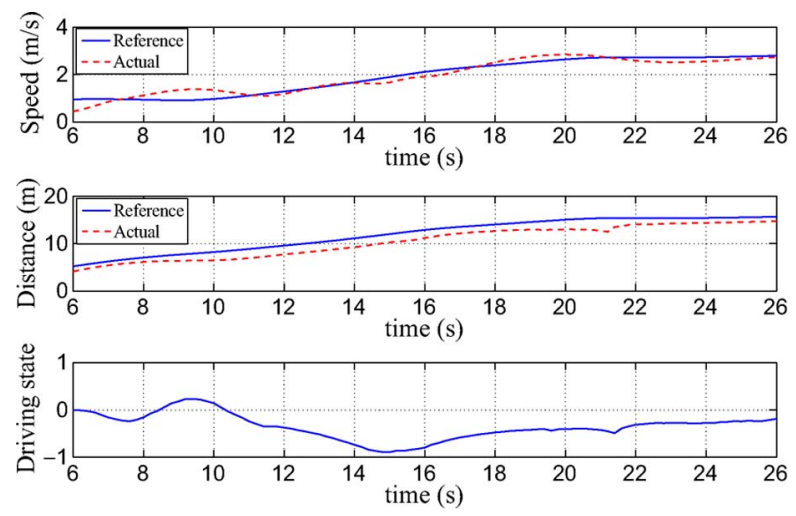

Fig. 12. Third vehicle in terms of right-of-way approaching the intersection.

Since the electric minibus has the right-of-way, information about traffic circumstances is neglected for this vehicle. Figs. 11-13 show the evolution of the second, third, and fourth vehicles, respectively, approaching the crossroad. The top plots show the actual speed (dashed line) and the reference speed (solid line). The middle plots show the actual distance between each vehicle and its leading car (dashed line) and the reference distance with respect to that car (solid line). In addition, the bottom plots show the Driving state, i.e., how the driver is managing the tradeoff between safety and traffic efficiency; this value is shown to the drivers in their head-up display.

All the vehicles started at the same time and at the same distance from the intersection. From that moment, the drivers try to follow the reference speed shown in their head-up display. One can appreciate how the second vehicle starts with a speed 

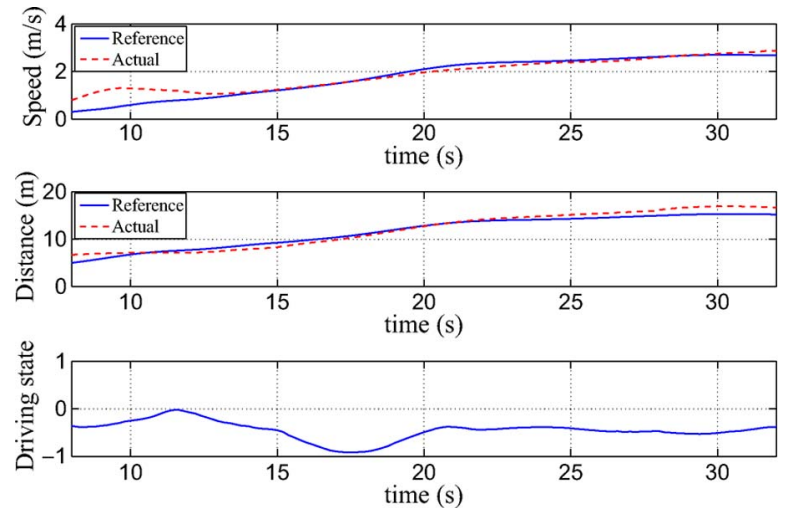

Fig. 13. Fourth vehicle in terms of right-of-way approaching the intersection.

greater than the reference value, and the collision probability significantly rises. At close to the time of $2 \mathrm{~s}$, the speed is readjusted, and the Driving state is reduced. During the rest of the experiment, the Driving state remains in the interval $[-1,0]$, and therefore, the driver is trying to follow the optimal behavior but giving priority to safety over traffic flow.

The behavior of the third and fourth vehicles is similar to that of the second. Once the drivers are capable of tracking the reference speed, the Driving state stays between -1 and 0 , particularly when the vehicle's speed is less than the reference value and the distance to the leading vehicle is greater than the reference.

Since the controller was adjusted to the behavior of real human drivers, it is very sensitive to minimal speed modifications, so that fluctuations in Driving state are to be expected, as is the case of a human driver approaching a cooperative traffic situation. Numerous trials were conducted to evaluate the behavior of the controller in the case of a sudden acceleration and/or deceleration of the vehicles involved in the maneuver. In all the cases, the Driving state remained coherent.

\section{CONCLUSION}

We have presented a V2I-based traffic management system with a twofold objective: First, our approach proposes a solution to the problem of regulating traffic flow in urban areas, in which different bottleneck situations may coexist. Second, it contributes to avoiding accidents by alerting the driver in advance of potential collisions. The system includes an intelligent controller that uses a reference safety distance and the appropriate speed as fuzzy inputs. The output sent to the driver is information on how the vehicle is being driven. In this paper, for the sake of simplicity, only information on longitudinal actions has been communicated.

The system has been tested in simulation and on a real test track. The results have shown it to perform well. This work constitutes a starting point for the development of a complete traffic control system. In particular, the authors would like to highlight that this paper presents the first results on how to manage four real vehicles approaching an intersection from different directions. Future work will study new challenges involving scenarios with traffic light control and lateral maneuvers.

\section{REFERENCES}

[1] A. Vahidi and A. Eskandarian, "Research advances in intelligent collision avoidance and adaptive cruise control," IEEE Trans. Intell. Transp. Syst., vol. 4, no. 3, pp. 143-153, Sep. 2003.

[2] A. Gonzalez, M. A. Garrido, D. F. Llorca, M. Gavilan, J. P. Fernandez, P. F. Alcantarilla, I. Parra, F. Herranz, L. M. Bergasa, M. A. Sotelo, and P. Revenga de Toro, "Automatic traffic signs and panels inspection system using computer vision," IEEE Trans. Intell. Transp. Syst., vol. 12, no. 2, pp. 485-499, Jun. 2011.

[3] R. Uzcategui and G. Acosta-Marum, "Wave: A tutorial," IEEE Commun. Mag., vol. 47, no. 5, pp. 126-133, May 2009.

[4] M. Yamamoto, K. Ohnishi, M. Seto, and K. Kamata, "Development of vehicle-infrastructure cooperative systems-Field operational test of hiroshima DSSS," in Proc. ITS World Congr., 2008.

[5] T. Aotani, S. Yamaoka, and T. Tajima, "Research development of driving safety support systems," in Proc. 41st SICE Annu. Conf., Aug. 2002, vol. 3, pp. 1792-1797.

[6] [Online]. Available: http://www.denso-europe.com/Vehicle-to-VehicleCommunications-1014820000000001.aspx

[7] [Online]. Available: http://www.cvisproject.org/

[8] [Online]. Available: http://www.safespot-eu.org/

[9] [Online]. Available: http://www.coopers-ip.eu/

[10] G. Toulminet, J. Boussuge, and C. Laurgeau, "Comparative synthesis of the 3 main european projects dealing with cooperative systems (cvis, safespot and coopers) and description of coopers demonstration site 4," in Proc. 11th Int. IEEE Conf. ITSC, Oct. 2008, pp. 809-814.

[11] L. Le, A. Festag, R. Baldessari, and W. Zhang, "Vehicular wireless shortrange communication for improving intersection safety," IEEE Commun. Mag., vol. 47, no. 11, pp. 104-110, Nov. 2009.

[12] I. Leontiadis, G. Marfia, D. Mack, G. Pau, C. Mascolo, and M. Gerla "On the effectiveness of an opportunistic traffic management system for vehicular networks," IEEE Trans. Intell. Transp. Syst., vol. 12, no. 4, pp. 1537-1548, Dec. 2011.

[13] A. Skordylis, "Efficient data propagation in traffic-monitoring vehicular networks," IEEE Trans. Intell. Transp. Syst., vol. 12, no. 3, pp. 680-694, Sep. 2011.

[14] S. A. Hosseini Tabatabaei, M. Fleury, N. N. Qadri, and M. Ghanbari, "Improving propagation modeling in urban environments for vehicular ad hoc networks," IEEE Trans. Intell. Transp. Syst., vol. 12, no. 3, pp. 705716, Sep. 2011.

[15] C.-L. Huang, Y. P. Fallah, R. Sengupta, and H. Krishnan, "Intervehicle transmission rate control for cooperative active safety system," IEEE Trans. Intell. Transp. Syst., vol. 12, no. 3, pp. 645-658, Sep. 2011.

[16] V. Milanés, J. Godoy, J. Pérez, B. Vinagre, C. González, E. Onieva, and J. Alonso, "V2I-Based architecture for information exchange among vehicles," in Proc. 7th Symp. Intell. Autonomous Veh., Sep. 2010.

[17] G. Bianchi, I. Tinnirello, and L. Scalia, "Understanding 802.11e contention-based prioritization mechanisms and their coexistence with legacy 802.11 stations," IEEE Netw., vol. 19, no. 4, pp. 28-34, Jul./Aug. 2005.

[18] D. Jiang and L. Delgrossi, "IEEE 802.11p: Towards an international standard for wireless access in vehicular environments," in Proc. Veh. Technol. Conf., 2008, pp. 2036-2040.

[19] R. Moraes, P. Portugal, F. Vasques, and R. Custódio, "Assessment of the ieee 802.11e EDCA protocol limitations when dealing with real-time communication," in Proc. EURASIP J. Wireless Commun. Netw., 2010, pp. 351 480-1-351480-14.

[20] Y. Wang, A. Ahmed, B. Krishnamachari, and K. Psounis, "IEEE 802.11p performance evaluation and protocol enhancement," in Proc. IEEE ICVES, 2008, pp. 317-322.

[21] A. Bohm and M. Jonsson, "Supporting real-time data traffic in safety critical vehicle-to-infrastructure communication," in Proc. 33rd IEEE Conf. $L C N$, 2008, pp. 614-621.

[22] S. Eichler, "Performance evaluation of the IEEE $802.11 \mathrm{p}$ wave communication standard," in Proc. IEEE 66th VTC, 2007, pp. 2199-2203.

[23] V. Milanés, J. Godoy, J. Villagrá, and J. Pérez, "Automated on-ramp merging system for congested traffic situations," IEEE Trans. Intell. Transp. Syst., vol. 12, no. 2, pp. 500-508, Jun. 2011.

[24] X. Lu, H. Tan, S. Shladover, and J. Hedrick, "Implementation of lontigudinal control algorithm for vehicle merging," in Proc. 5th Int. Symp. Adv. Veh. Control, Aug. 2000, pp. 25-32.

[25] M. Persson, F. Botling, E. Hesslow, and R. Johansson, "Stop and go controller for adaptive cruise control," in Proc. IEEE Int. Conf. Control Appl., 1999, vol. 2, pp. 1692-1697.

[26] K. Yi and I. Moon, "A driver-adaptive stop-and-go cruise control strategy," in Proc. IEEE Int. Conf. Netw. Sens. Control, 2004, vol. 1, pp. 601-606. 
[27] M. Brackstone and M. McDonald, "Car- following: A historical review," Transp. Res. F, vol. 2, no. 4, pp. 181-196, Dec. 2000

[28] O. N. T. Hiraoka, T. Kunimatsu, and H. Kumamoto, "Modeling of driver following behavior based on minimum-jerk theory," in Proc. 12th World Congr. ITS, 2005, pp. 6-10.

[29] J. Martinez and C. C. de Wit, "A safe longitudinal control for adaptive cruise control and stop-and-go scenarios," IEEE Trans. Control Syst. Technol., vol. 15, no. 2, pp. 246-258, Mar. 2007.

[30] J. Villagrá, V. Milanés, J. Pérez, and C. Gonzalez, "Model-free control techniques for stop \& go systems," in Proc. 13th Intell. Transp. Syst. Conf., Sep. 2010, pp. 1899-1904.

[31] V. Milanés, J. Villagrá, J. Godoy, and C. González, "Comparing fuzzy and intelligent pi controllers in stop-and-go manoeuvres," IEEE Trans. Control Syst. Technol., pp. 1-9, Apr. 2011, to be published, DOI: 10.1109/TCST.2011.2135859.

[32] R. Precup and H. Hellendoorn, "A survey on industrial applications of fuzzy control," Comput. Ind., vol. 62, no. 3, pp. 213-226, Apr. 2011.

[33] I. Tejado, V. Milanés, J. Villagrá, J. Godoy, S. Hassan, and B. Vinagre, "Low speed control of an autonomous vehicle by using a fractional pi," in Proc. 18th IFAC World Congr., Aug. 2011, pp. 15 025-15030.

[34] J. Perez, V. Milanes, and E. Onieva, "Cascade architecture for lateral control in autonomous vehicles," IEEE Trans. Intell. Transp. Syst., vol. 12, no. 1, pp. 73-82, Mar. 2011.

[35] V. Milanés, J. Pérez, E. Onieva, and C. González, "Controller for urban intersections based on wireless communications and fuzzy logic," IEEE Trans. Intell. Transp. Syst., vol. 11, no. 1, pp. 243-248, Mar. 2010.

[36] J. Godoy, V. Milanés, J. Perez, J. Villagrá, and C. Gonzalez, "Power electric aiding controller for automated bus stopping," in Proc. CPE, 2011, pp. 330-335.

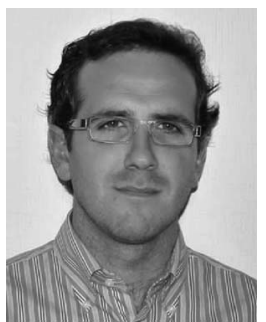

Vicente Milanés received the B.E. and M.E. degrees in electronic engineering from the University of Extremadura, Badajoz, Spain, in 2002 and 2006, respectively, and the Ph.D. degree in electronic engineering from Alcala University, Madrid, Spain, in 2010.

Since 2006, he has been with the Spanish National Research Council, Centre for Automation and Robotics, Madrid. His research interests include autonomous vehicles, fuzzy-logic control, intelligent traffic and transport infrastructures, and vehicle-infrastructure cooperation.

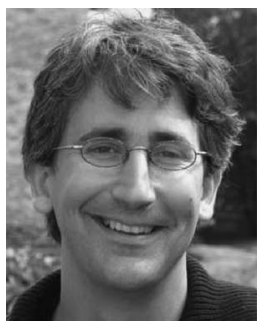

Jorge Villagrá received the M.E. degree in electrical engineering from the Universidad Politécnica of Madrid, Madrid, Spain, in 2002 and the Ph.D. degree from the Ecole des Mines de Paris, Paris, France, in 2006.

From 2006 to 2009, he was a Research Assistant with INRIA-Roquencourt, France, and a Visiting Professor with the University Carlos III, Madrid. $\mathrm{He}$ is currently a Research Fellow with the AUTOPIA Program, Centre for Automation and Robotics, Madrid. His research interests include nonlinear and optimal control and nonlinear estimation for intelligent transportation systems.

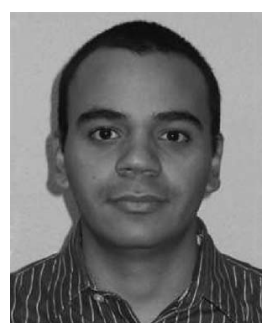

Jorge Godoy was born in Maracay, Venezuela, in 1986. He received the Electronic Engineer degree from the Universidad Simón Bolívar, Caracas, Venezuela, in 2008 . He is currently working toward the M.E. degree in automation and robotics with the Universidad Politécnica de Madrid, Madrid, Spain.

Since 2009, he has been doing research on autonomous vehicles with the UPM-CSIC Centre for Automation and Robotics, Madrid. His research interests include fuzzy-logic control and intelligent transportation systems.

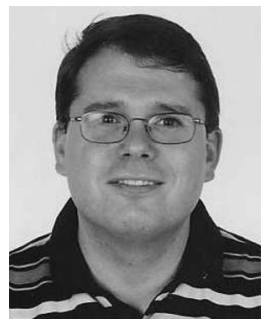

Javier Simó was born in Valladolid, Spain, in 1970. He received the B.E., M.E., and Ph.D. degrees in telecommunications engineering from the Universidad Politécnica de Madrid, Madrid, Spain, in 1996, 2005, and 2007, respectively.

Since 2005, he has been an Associate Professor with the Department of Signal Theory and Communications, Universidad Rey Juan Carlos, Madrid. His research interests include vehicle-to-infrastructure and vehicle-to-vehicle communications using Wireless Access in Vehicular Environments (WiMAX) and broadband rural wireless networks.

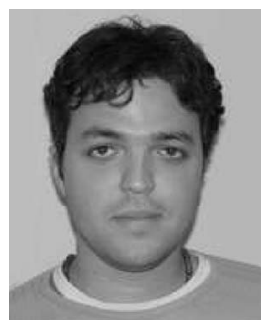

Joshué Pérez was born in Coro, Venezuela, in 1984. $\mathrm{He}$ received the B.E. degree in electronic engineering from the Universidad Simón Bolívar, Caracas, Venezuela, in 2007 and the M.E. degree in systems engineering and automatic control from the University Complutense of Madrid, Madrid, Spain, in 2009. $\mathrm{He}$ is currently working toward the Ph.D. degree with the Centre for Automation and Robotics, Madrid.

His research interests include fuzzy logic, modeling, control, and cooperative maneuvers among autonomous vehicles.

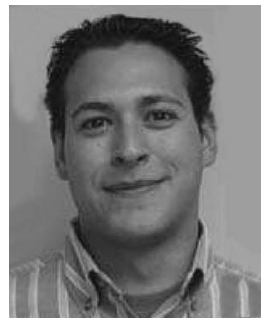

Enrique Onieva received the B.E. and M.E. degrees in soft computing and intelligent systems from the University of Granada, Granada, Spain, in 2006 and 2008, respectively.

Since 2007, he has been with the Industrial Computer Science Department (currently the Centre of Automation and Robotics), Madrid, Spain. His research interests include autonomous vehicles, fuzzylogic control, and intelligent transportation systems. 